\title{
X-ray powder diffraction and electron single crystal diffraction - two techniques for structure analysis of nanocrystals
}

\author{
T. E. Gorelik \\ Ulm University, Central Facility for Electron Microscopy, Electron Microscopy Group of Materials Science (EMMS), \\ Albert Einstein Allee 11, 89081 Ulm, Germany \\ tatiana.gorelik@uni-ulm.de
}

X-ray powder diffraction and electron single crystal diffraction, although having very different methodologies in their cores, target the same material, and can deliver complimentary information for the structure characterization.

$\mathrm{X}$-ray powder diffraction is a well-established technique; its performance can be exemplified by several impressive highlights [1-3]. A structure analysis with powder X-ray diffraction runs thorough three main steps - (i) indexing of the powder profile, (ii) structure solution, and (ii) structure refinement. The first step represents the bottleneck for the whole procedure, being associated with the inherent problem of the powder method - projection of all reflections onto a single axis. The most difficult cases represent polyphasic samples, large unit cell volumes, and low symmetry structures.

Electron diffraction method, being able to address nanocrystals individually, allows to collect 3D single crystal data from crystals with the size down to tens of nanometres [4]. A 3D reconstruction of the reciprocal space immediately delivers information on the unit cell metric. The inherent problems of electron diffraction appear at later stages when quantification of reflection intensities is required. The strong interaction of electrons with matter gives rise to multiple scattering, which modifies intensities of reflections in a complex manner. Recently, methods for dynamical structure refinement became available [5]; still the multiple scattering contribution cannot be accounted for during the structure solution (model building) step.

In this light, an obvious beneficial combination of two techniques is the transfer of unit cell parameters, determined from electron diffraction to powder X-ray data for subsequent structure solution and refinement. This workflow will be demonstrated by examples. Beyond this combination, analysis of diffuse scattering by the two methods will be presented, and combined analysis of total scattering for PDF calculation will be discussed.

[1] Vella-Zarb, L., Baisch, U., Dinnebier, R. E. (2013). J. Pharm. Sci., 102, 674.

[2] Schlesinger, C., Bolte, M. and Schmidt, M. U. (2019). Z. Kristallogr. 234, 257.

[3] Spiliopoulou, M. Karavassili, F. Triandafillidis, D.-P. Valmas, A. Fili, S. Kosinas, C. Barlos, K. Barlos, K. K. Morin, M. Reinle-Schmitt, M. L. Gozzo F. and Margiolaki, I. (2021). Acta Cryst. A77.

[4] Gemmi, M., Mugnaioli, E., Gorelik, T.E., Kolb, U., Palatinus, L., Boullay, P., Hovmöller, S., Abrahams, J.P. (2019). ACS Cent. Sci. 5 , 1315.

[5] Palatinus, L. Brázda, P. Jelínek, M. Hrdá J., Steciuk, G. Klementová M. (2019). Acta Cryst., B75, 512.

Keywords: 3D electron diffraction; powder X-ray diffraction; nanocrystals; diffuse scattering; PDF 